\title{
A Power Efficiency Improvement Technique for A Bi-Directional Dual Active Bridge DC-DC Converter at light load
}

\author{
Mika Takasaki, Yoichi Ishizuka, Tamotsu Ninomiya \\ Nagasaki University \\ Graduate school of engineering \\ Nagasaki, Japan \\ \{bb52112218@cc., isy2@,ninomiya@\}nagasaki-u.ac.jp
}

\author{
Yutaka Furukawa \\ Koga System Works \\ Saga, Japan \\ frkw@kogasw.com
}

\author{
Toshiro Hirose \\ Nishimu Electronics Industries Co.,Ltd. \\ Saga Plant \\ Saga, Japan \\ hirose@nishimu.co.jp
}

\begin{abstract}
Recently, the bi-directional dc-dc converter has been focused on because of the huge demand for diversification of power supply network including battery. The dual active bridge (DAB) dc-dc converter is one of the most popular circuits for bi-directional applications because of its simple structure. However, power efficiency at light load is the intrinsic problem of a bi-directional DAB DC-DC converter. In this paper, the simple solution with digital operation for the problem is proposed and experiments are performed with $1 \mathrm{~kW}$ system. This method can reduce a switching surge without other circuits such as snubber and improve power efficiency at light load. Therefore it can reduce loss of switching surge and, improve power efficiency. From the results, $37 \%$ maximum power efficiency improvement at light load is confirmed. Furthermore, this method is capable of control in the conventional method in the heavy load range. Consequently, it is possible to reduce the switching surge and realize high power efficiency in a wide load range.
\end{abstract}

\section{INTRODUCTION}

Recently, the bi-directional dc-dc converter has been focused on because of the huge demand for diversification of power supply network including battery. The DAB dc-dc converter is one of the most popular circuits for bidirectional applications because of its simple structure [1-6]. However, a switching surge and power efficiency at light load condition is the intrinsic problem [4].

Some research have been done to solve the problem, for instance, use of resonant type converter with snubber circuit [1], silicon carbide $(\mathrm{SiC})$ power device and new magnetic materials [2], Quasi-ZCS operation with LC filter [3], and converter linked through superposition in additive polarity in series[4].

This paper proposes a simple solution for power efficiency improvement with digital operation. This method can improve power efficiency due to reduce a switching loss without adding other circuits such as snubber.

\section{Conventional Operation Of a DAB DC-DC CONVERTER}

Fig. 1 shows the circuit schematic of the basic DAB dcdc converter. Fig. 2 shows the operating waveforms with the conventional operation [5]. In the conventional operation, the output power is operated by the phase-shift shown as $\varphi$ between the primary voltage $v_{\mathrm{P}}$ and secondary voltage $v_{\mathrm{S}}$ of transformer. Fig. 3 shows the phasor diagram. $V_{\mathrm{P}}, V_{\mathrm{S}}, V_{\mathrm{L}}$, and $I$ are phasor symbols for $v_{\mathrm{P}}, v_{\mathrm{S}}, v_{\mathrm{L}}, i$, respectively. When

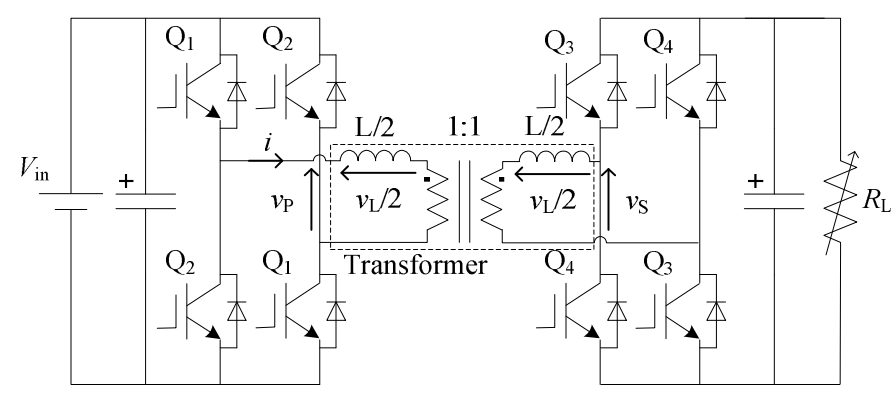

Figure 1. The circuit schematic of DAB dc-dc converter. 


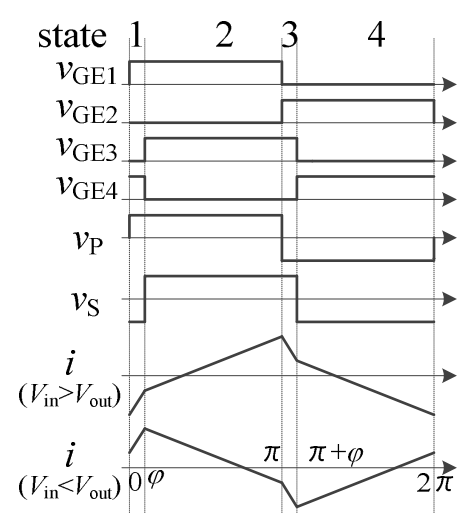

(a)

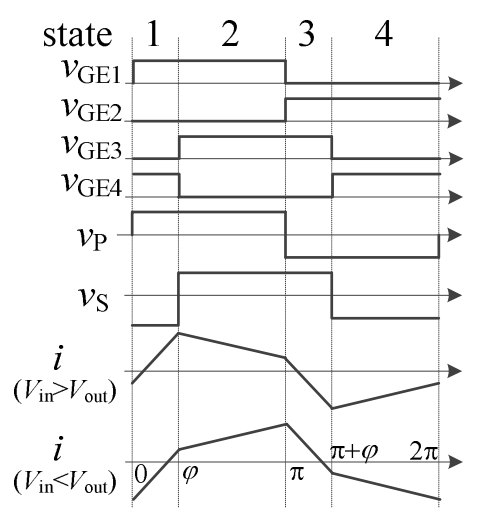

(b)

Figure 2. Conventional operating waveform:

(a) light load; (b) heavy load.

$V_{\mathrm{S}}$ is lagging $V_{\mathrm{P}}$ in power running mode (Fig. 3 (a) and (b)), and when $V_{\mathrm{S}}$ is leading $V_{\mathrm{P}}$, it is operated in power regenerative mode (Fig. 3 (c)).

The output power $P_{\mathrm{o}}$ can be obtained as

$$
P_{o}=\frac{V_{\text {in }} V_{o u t}}{\omega L} \varphi\left(1-\frac{\varphi}{\pi}\right) .
$$

The output power can be controlled with the phase difference $\varphi$. The waveform of the current $i$ is changed by the load condition. In this paper, current $i$ crossed the zero line in the state 2 is defined as a light load, and current $i$ crossed the zero line in the state 1 is defined as a heavy load as shown in Fig. 2.

\section{INTRINSIC SURGE Problem OF A DAB DC-DC CONVERTER}

Well known problem of a DAB DC-DC converter is switching surge in the light condition. It is caused by the reverse recovery effect of the diode. Fig. 4 shows $\varphi-P_{\mathrm{o}}$. The switching surge occurs at hard switching range of this figure.

Fig. 5 shows the generation mechanism of switching surge when $V_{\text {in }}>V_{\text {out }}$. The surge voltage occurs in the transition from state 1 (3) to state 2 (4), repeatedly. $C_{\mathrm{d}}$ is the parasitic capacitance of diode which is connected in parallel with the ideal diode, and $L_{\text {wire }}$ is parasitic reactance. At the light load condition, the diodes $\mathrm{D}_{4}$ is conducting in state 1. Then the switches $Q_{3}$ is turned on when state changes from state 1 to state 2 . At this instantaneous moment, the

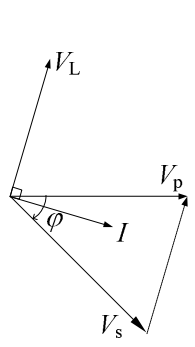

(a)

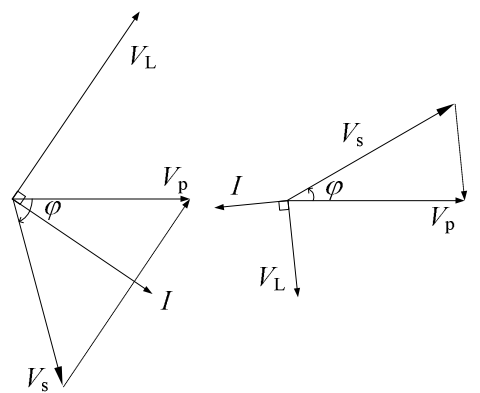

(b) (c)
Figure 3. Phasor diagram[1]: (a) power running mode (light load); (b) power running mode (heavy load); (c) power regenerative mode.

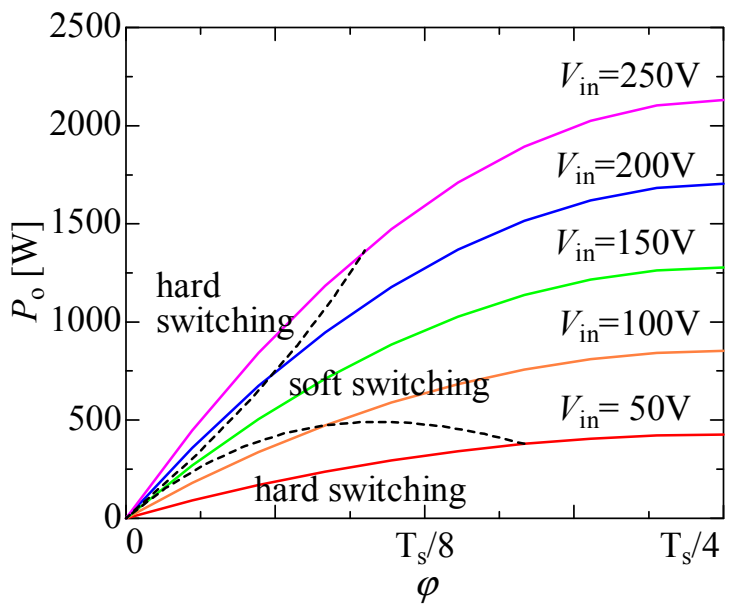

Figure 4. $\varphi$ - $P_{0}[1]$.

diode $\mathrm{D}_{4}$ is switched from a forward bias condition to a reverse bias condition immediately. And the switching surge is occurred with the resonance of $C_{\mathrm{d}}$ and $L_{\text {wire }}$ due to reverse recovery phenomenon. With the same reason, when $V_{\text {in }}<V_{\text {out }}$, the surge occurs in the transition from state 2 (4) to state 3 (1) on the primary side.

Commonly, to protect the switches from the switching surge, snubber circuit are applied [2]. However, the power loss at the snubber circuit can't be ignored at the light load condition. The other way, the resonant converter type is also popular, but the additional components are needed.

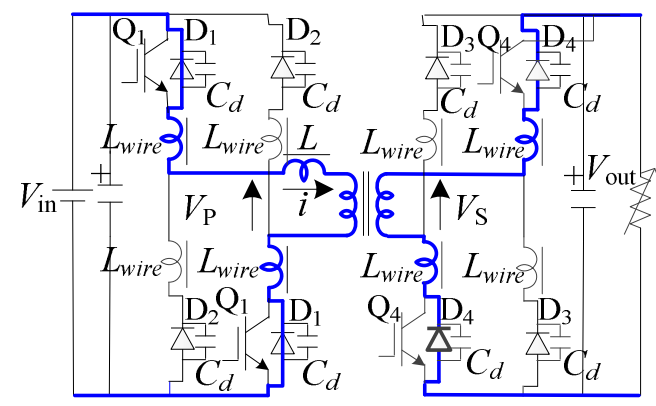

(a) state 1

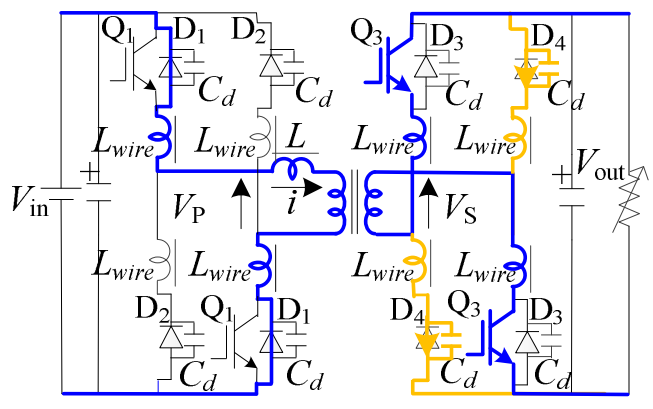

(b) state 2

Figure 5. The generation mechanism of switching surge. 


\section{PROPOSED OPERATION METHOD}

We have proposed the software-based compensation method for basic DAB bi-directional dc-dc converter which can be reduce the switching surge at the light load, without any of additional circuits such as the snubber circuits or resonant circuits [7]. Fig. 6 shows idealized waveform of the proposed operating method. With this method, it can easily change alternately buck mode and boost mode operation. When $V_{\text {in }}<$ $V_{\text {out }}$, as it can be seen from the waveforms, the direction of primary side current of transformer $i$ during each on-time of $\mathrm{Q}_{1}$ and $\mathrm{Q}_{2}$, is restricted to avoid the crossing the zero line. Due to the restriction of the change of the direction of the current, the zero-current-switching-on can be realized for $\mathrm{Q}_{1}$ and $\mathrm{Q}_{2}$. The ideal analysis has been done for each of buck mode and boost mode operation, respectively. This converter has six operational states in one switching period for each of the buck and boost mode operation, respectively. The elements are treated as ideal in equivalent circuit.

The detailed description of the ideal circuit is revealed in a previous paper [7]. Therefore, only the results are shown in this paper.

\section{A. Buck Mode Operation in light load}

In buck mode, the primary side switches $\mathrm{Q}_{1}$ and $\mathrm{Q}_{2}$ are turned-on twice in the period. Firstly, $\mathrm{Q}_{1}$ and $\mathrm{Q}_{2}$ are turn-on at $t=0$ and $T_{\mathrm{s}} / 2$. Secondly, they are turn-off at $t=A$ and $T_{\mathrm{s}} /$ $2+A$. Thirdly, they are turn-on at $t=\varphi$ and $T_{\mathrm{s}} / 2+\varphi$. Fourthly, they are turn-off at $t=T_{\mathrm{s}} / 2$ and $T_{\mathrm{s}}$.

$A$ is calculated as

$$
A=\frac{V_{i}-V_{o}}{V_{i}+V_{o}}\left(\frac{1}{2} T_{s}-\varphi\right) .
$$

\section{B. Boost Mode Operation in light load}

In boost mode, the secondary side switches $\mathrm{Q}_{3}$ and $\mathrm{Q}_{4}$ are turned-on twice in the period. Firstly, $\mathrm{Q}_{3}$ and $\mathrm{Q}_{4}$ are turn-on at $t=\varphi$ and $T_{\mathrm{s}} / 2+\varphi$, respectively. Secondly, they are turn-

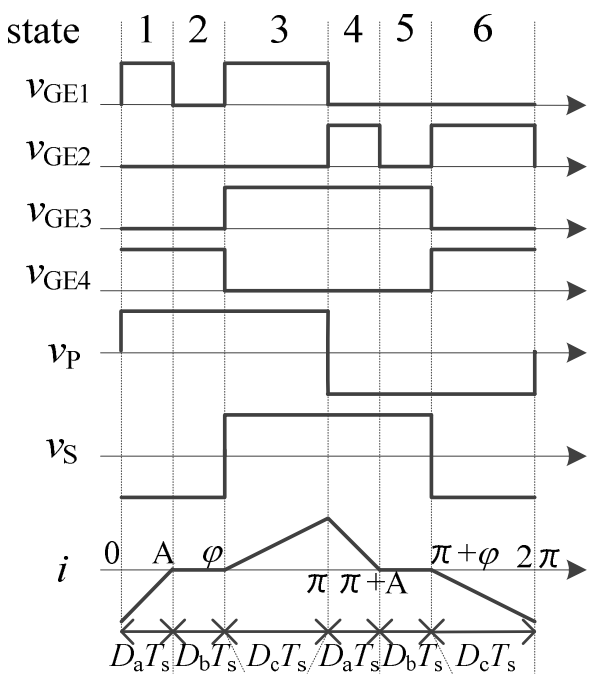

(a) buck mode off at $t=B$ and $T_{\mathrm{s}} / 2+B$. Thirdly, they are turn-on at $t=T_{\mathrm{s}} /$ 2 and 0 . Fourthly, they are turn-off at $t=T_{\mathrm{s}} / 2+\varphi$ and $B$.

$B$ is calculated as

$$
B=\frac{2 V_{o}}{V_{o}-V_{i}} \varphi
$$

\section{Output power control in light load}

The ideal analysis for both of buck and boost mode operation can be done in uniformly. For the ideal analysis result, the output power $P_{\mathrm{o}}$ can be obtained as

$$
P_{o}=\frac{2 X^{2}}{T_{s} L} \cdot\left|\frac{V_{i}+V_{o}}{V_{i}-V_{o}}\right| \cdot V_{i} V_{o} .
$$

In buck mode, $X=A$, and in boost mode, $X=\varphi$.

\section{Output Power control in heavy load}

In the light load, with the output power increasing, the periods of which all switches turned OFF $(A \sim \varphi, \pi+A \sim \pi+\varphi$, $B \sim \pi, \pi+B \sim 2 \pi$ ) becomes shorter. The periods, equal to zero seconds, it is the boundary between light load and heavy load. Therefore, in the heavy load condition, the only conventional phase-shift operation is active. From the results, it can be seen that it is possible to control the output power seamlessly despite of the load condition. Relationship $\varphi$ and $P_{\mathrm{o}}$ of conventional and proposed operation is shown in Fig. 7.

\section{E. Pulse generating method}

Fig. 8 and Fig. 9 show the generating mechanism of proposed drive signal. As mentioned above, the gate signal is the combination of the phase shift signal and the masked signal. The mask width is calculated and controlled by Eq. (2) and (3), respectively.

\section{LOSS INCLUDED ANALYSIS OF CONVENTIONAL OPERATION}

The loss included analysis of conventional operation is shown in a previous paper [8]. Therefore, only the results are

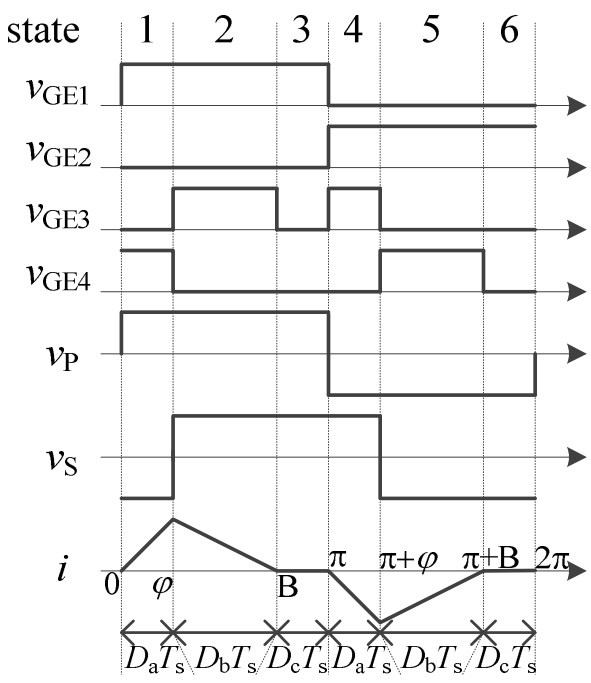

(b) boost mode

Figure 6. Idealized wave form for proposed operating at the light load: (a) buck mode; (b) boost mode. 


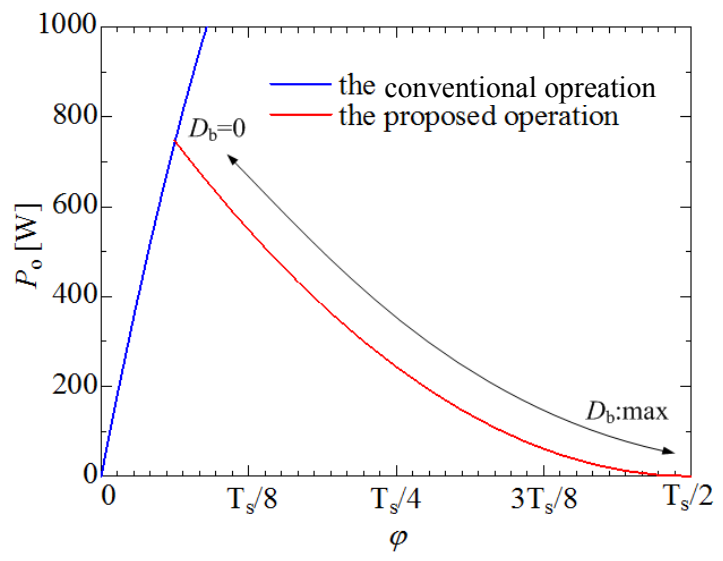

(a) buck mode

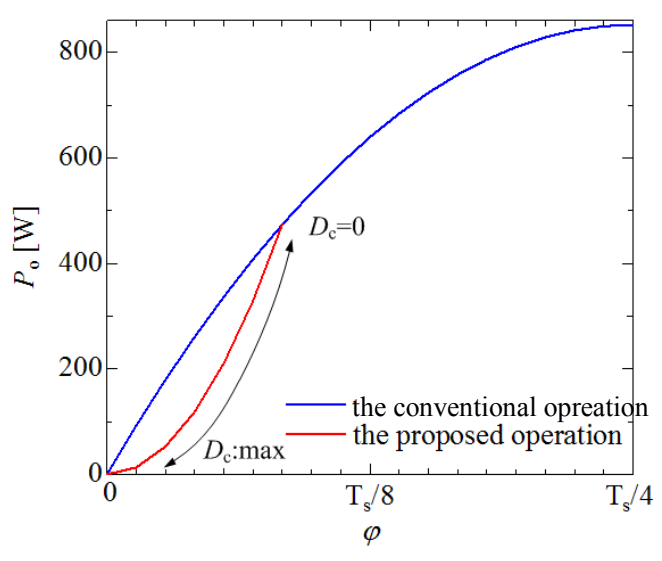

(b) boost mode

Figure 7. $\varphi-P o$ (conventional operation and proposed operation).

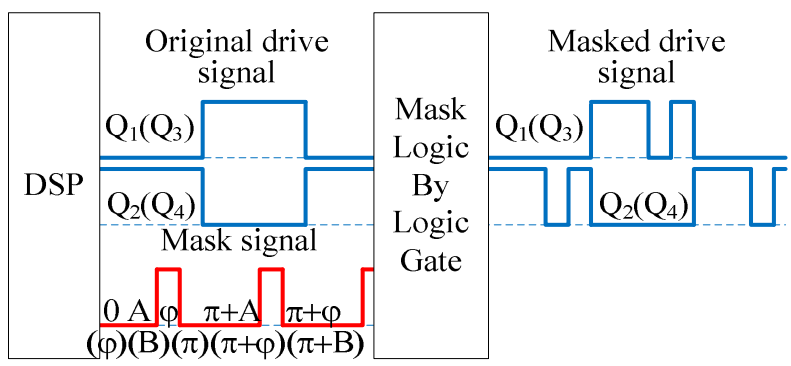

Figure 8. Masked drive signal generating mechanism. shown in this paper. Output power including the loss is

$$
P_{o}=\frac{V_{\text {in }} V_{\text {out }}}{2} \varphi\left(1-\frac{\varphi}{\pi}\right)\left[\frac{V_{o} / V_{i}+1}{\omega L+r_{\text {loss }} \varphi}-\frac{V_{o} / V_{i}-1}{\omega L+r_{\text {loss }}(\pi-\varphi)}\right] .
$$

\section{LOSS INCLUDED ANALYSIS OF PROPOSED OPERATION}

To analyze the characteristics of the circuit, Extended State-Space Averaging Method [9] is applied. The analysis has been done for each of buck mode and boost mode operation, respectively. In order to simplify the loss analysis, loss is defined as $r_{\text {loss. }}$.

\section{A. Buck Mode Operation}

Equivalent circuits corresponding to each state in buck mode operation are shown in Fig. 10, where $\hat{v}_{o}$ is the lowfrequency component of $V_{\mathrm{o}} . D_{\mathrm{a}}=A-0, D_{\mathrm{b}}=\varphi-A, D_{\mathrm{c}}=\pi$ $\varphi$ in Fig. 6 (a). For ease of analysis, the calculation has been performed in a half of the switching period because of the symmetric behavior of the circuit.

For analysis, solving for $i_{\mathrm{L}}$ and $i_{\mathrm{c}}$, for $0 \leq t \leq A$ (state 1 )

$$
\begin{gathered}
i_{L}=\frac{\left(V_{i}+\hat{v}_{0}\right) t}{L+r_{\text {loss }} t}+\frac{L}{L+r_{\text {loss }} t} i(0) \\
i_{C}=-i_{L}-\frac{\hat{v}_{o}}{R_{L}}
\end{gathered}
$$

for $A \leq t \leq \varphi$ (state 2)

$$
i_{L}=0
$$

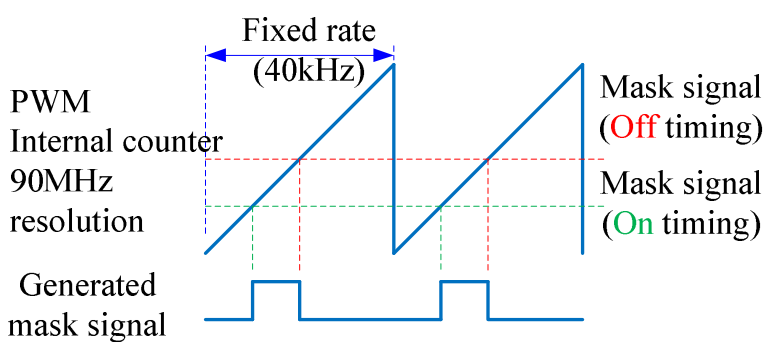

Figure 9. Mask signal generating mechanism by PWM peripheral in DSP.

$$
i_{C}=-i_{L}-\frac{\hat{v}_{o}}{R_{L}}
$$

for $\varphi \leq t \leq \pi$ (state 3$)$

$$
\begin{gathered}
i_{L}=\frac{\left(V_{i}-\hat{v}_{0}\right)\left\{t-\left(D_{a}+D_{b}\right) T_{s}\right\}}{L+r_{\text {loss }}\left\{t-\left(D_{a}+D_{b}\right) T_{s}\right\}} \\
i_{C}=i_{L}-\frac{\hat{v}_{o}}{R_{L}} .
\end{gathered}
$$

From Fig. 6, it is clear that $D_{\mathrm{a}}+D_{\mathrm{b}}+D_{\mathrm{c}}=1 / 2$, $i_{\mathrm{L}}(0)=-i_{\mathrm{L}}(\pi)$ and $i_{\mathrm{L}}(A)=0$. Using the preceding relationships,

$$
i_{L}(0)=-i_{L}(\pi)=-\frac{\left(V_{i}+\hat{v}_{0}\right) D_{a} T_{s}}{L}
$$

and

$$
D_{c}=\frac{V_{i}+\hat{v}_{o}}{V_{i}-\hat{v}_{o}-2 D_{a} T_{s} \hat{v}_{o} r_{\text {loss }} / L} D_{a} .
$$

The average value of $v$ in each state is calculated with

$$
\begin{gathered}
i_{c_{-} \text {avel }}=-\frac{1}{2} \frac{\left(V_{i}+\hat{v}_{0}\right) D_{a} T_{s}}{L}-\frac{\hat{v}_{o}}{R_{L}} \\
i_{c_{-} a v e 2}=-\frac{\hat{v}_{o}}{R_{L}} \\
i_{c_{-} \text {ave3 }}=-\frac{1}{2} \frac{\left(V_{i}+\hat{v}_{0}\right) D_{a} T_{s}}{L}-\frac{\hat{v}_{o}}{R_{L}} .
\end{gathered}
$$

Hence, 


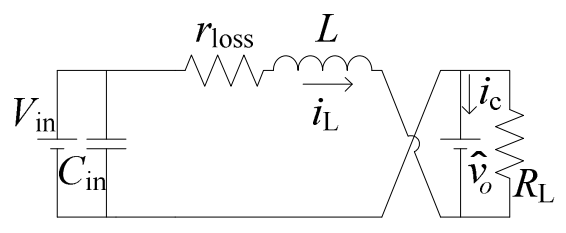

(a) state 1

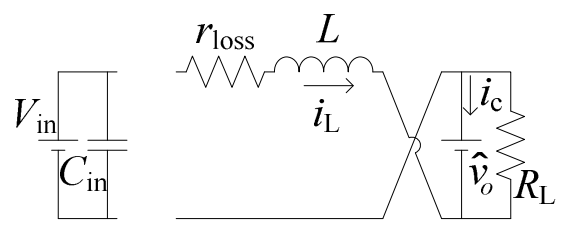

(b) state 2

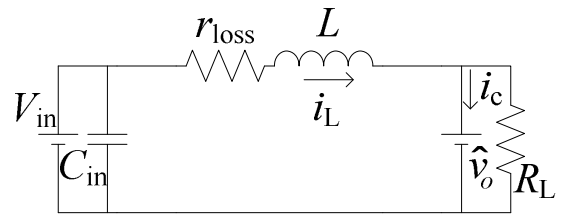

(c) state 3

Figure 10. Equivalent circuit of buck mode operation: (a) state 1; (b) state 2; (c) state 3 .

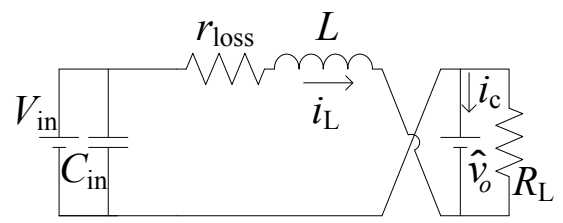

(a) state 1

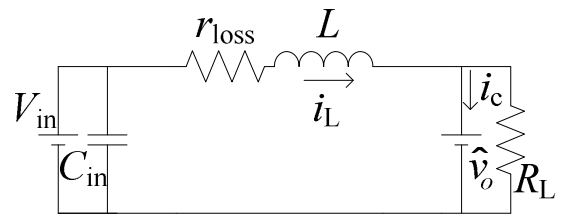

(b) state 2

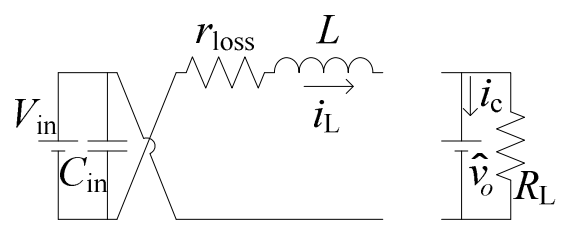

(c) state 3

Figure 11. Equivalent circuit of boost mode operation: (a) state 1 ; (b) state 2 ; (c) state 3 .

$$
\begin{aligned}
\overline{i_{c}} & =2\left(i_{c_{-} a v e 1} \times D_{a}+i_{c_{-} a v e 2} \times D_{b}+i_{c_{-} a v e 3} \times D_{c}\right) \\
& =\frac{2 D_{a}^{2} T_{s}}{L} \cdot \frac{\left(V_{i}+\hat{v}_{o}\right)\left(V_{i}-\hat{v}_{o} D_{a} T_{s} r_{\text {loss }} / L\right)}{\left(V_{i}-\hat{v}_{o}\right)}-\frac{\hat{v}_{o}}{R_{L}} .
\end{aligned}
$$

The results of static characteristics are obtained by letting $d \hat{v}_{o} / d t=0$, therefore

$$
\begin{gathered}
\overline{i_{c}}=C \frac{d \hat{v}_{o}}{d t}=0 \\
P_{o}=\frac{2 D_{a}^{2} T_{s}}{L} \cdot \frac{\left(V_{i}+V_{o}\right)\left(V_{i} V_{o}-V_{o}^{2} D_{a} T_{s} r_{\text {loss }} / L\right)}{\left(V_{i}-V_{o}\right)} .
\end{gathered}
$$

Using $D_{a} T_{s}=A$,

where

$$
P_{o}=\frac{2 A^{2}}{T_{s} L} \cdot \frac{\left(V_{i}+V_{o}\right)\left(V_{i} V_{o}-V_{o}^{2} D_{a} T_{s} r_{\text {loss }} / L\right)}{\left(V_{i}-V_{o}\right)}
$$

$$
A=\frac{\left(V_{i}-V_{o}\right)\left(T_{s} / 2-\varphi\right)}{V_{i}+V_{o}+2 V_{o}\left(T_{s} / 2-\varphi\right) r_{l o s s} / L} .
$$

\section{B. Boost Mode Operation}

Equivalent circuits corresponding to each state in boost mode operation are shown in Fig. 11 For analysis, equation is formularized for each state. $D_{\mathrm{a}}=\varphi-0, D_{\mathrm{b}}=B-\varphi, D_{\mathrm{c}}=\pi$ - $B$ in Fig. 6 (b).

For $0 \leq t \leq \varphi($ state 1$)$

$$
\begin{gathered}
i_{L}=\frac{\left(V_{i}+\hat{v}_{0}\right) t}{L+r_{\text {loss }} t} \\
i_{C}=-i_{L}-\frac{\hat{v}_{o}}{R_{L}}
\end{gathered}
$$

for $\varphi \leq t \leq B$ (state 2)

$$
\begin{gathered}
i_{L}=\frac{\left(V_{i}-\hat{v}_{0}\right)\left(t-D_{a} T_{s}\right)}{L+r_{\text {loss }}\left(t-D_{a} T_{s}\right)}+\frac{L}{L+r_{\text {loss }}\left(t-D_{1} T_{s}\right)} i\left(D_{a} T_{s}\right) \\
i_{C}=i_{L}-\frac{\hat{v}_{o}}{R_{L}}
\end{gathered}
$$

for $B \leq t \leq \pi$ (state 3 )

$$
\begin{gathered}
i_{L}=0 \\
i_{C}=-\frac{\hat{v}_{o}}{R_{L}} .
\end{gathered}
$$

From Fig. 6, it is clear that $D_{\mathrm{a}}+D_{\mathrm{b}}+D_{\mathrm{c}}=1 / 2$, and $i_{\mathrm{L}}(B)$ $=0$. Using the preceding relationships

$$
D_{b}=-\frac{\left(V_{i}+\hat{v}_{o}\right)}{\left(V_{i}-\hat{v}_{o}\right)\left(1+D_{a} T_{s} r_{\text {loss }} / L\right)} D_{a} .
$$

Hence,

$$
\overline{i_{c}}=-\frac{2 D_{a}^{2} T_{s}}{L} \cdot \frac{\left(V_{i}+\hat{v}_{o}\right)}{\left(V_{i}-\hat{v}_{o}\right)} \cdot \frac{\left\{V_{i}+\left(V_{i}-\hat{v}_{o}\right) D_{a} T_{s} r_{\text {loss }} / 2 L\right\}}{\left(1+D_{a} T_{s} r_{\text {loss }} / L\right)^{2}}-\frac{\hat{v}_{o}}{R_{L}} .
$$

The results of static characteristics are obtained by letting $d \widehat{v}_{o} / d t=0$, therefore

$P_{o}=\frac{2\left(D_{a} T_{s}\right)^{2}}{T_{s} L} \cdot \frac{\left(V_{i}+V_{o}\right)}{\left(V_{i}-V_{o}\right)} \cdot \frac{\left\{V_{i} V_{o}+V_{o}\left(V_{i}-V_{o}\right) D_{a} T_{s} r_{\text {loss }} / 2 L\right\}}{\left(1+D_{a} T_{s} r_{\text {loss }} / L\right)^{2}}$.

Using $D_{a} T_{s}=\varphi$

$$
P_{o}=\frac{2 \varphi^{2}}{T_{s} L} \cdot \frac{\left(V_{i}+V_{o}\right)}{\left(V_{i}-V_{o}\right)} \cdot \frac{\left\{V_{i} V_{o}+V_{o}\left(V_{i}-V_{o}\right) \varphi r_{\text {loss }} / 2 L\right\}}{\left(1+\varphi r_{\text {loss }} / L\right)^{2}} .
$$


$B$ is calculated as

$$
B=\left(D_{a}+D_{b}\right) T_{s}=\frac{-2 V_{o}-\left(V_{i}-V_{o}\right) \varphi r_{\text {loss }} / L}{\left(V_{i}-V_{o}\right)\left(1+D_{a} T_{s} r_{\text {loss }} / L\right)} \varphi .
$$

\section{EXPERIMENTAL RESULTS}

In order to select the value of $r_{\text {loss }}$, we perform some experiments with the prototype circuit. The main circuit is DAB dc-dc converter without additional circuits like snubber circuit. We had closed loop operation experiments with DSP TI TMS320F28335. Experimental parameters are shown in Table I. Dead time of each switch is $1 \mu \mathrm{s}$.

\section{A. Surge Reduction}

Fig. 12 shows the waveform of the corrector-emitter voltage and the corrector current of the low voltage side bridge of the buck converter. Fig. 12(a) shows the result of the conventional operation and Fig. 12(b) shows the result of the proposed operation. Comparing with these results, it can be seen that $99 \%$ of voltage surges and $100 \%$ of current serge of reduction.

Fig. 13 shows the waveform of the boost converter. Comparing with these results, it can be seen that $99 \%$ of voltage surges of reduction and $100 \%$ of current serge of reduction.

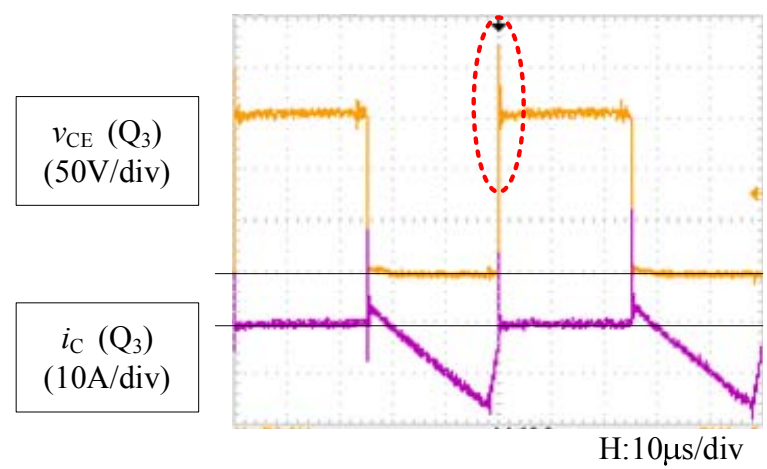

(a) the conventional operation

\section{B. Power efficiency}

Fig. 14 and Fig. 15 show the comparison of the current waveforms of $i_{\mathrm{L}}$ in the condition of the regulated output voltage. From the results, it can be seen that the amount of the $i_{\mathrm{L}}$

TABLE I. SPECIFICATION OF DAB DC-DC CONVERTER

\begin{tabular}{|c|c|c|}
\hline Item & Symbol & Specification \\
\hline \multicolumn{3}{|l|}{ Transformer } \\
\hline 1) Turns ratio & $A$ & $1: 1$ \\
\hline 2) Leakage inductance(primary-referred) & $L$ & $110 \mu \mathrm{H}$ \\
\hline \multicolumn{3}{|l|}{ Converter } \\
\hline 1) Rated output power & $P_{\mathrm{o}}$ & $1 \mathrm{~kW}$ \\
\hline 2) Rated input direct voltage & $V_{\text {in }}$ & $150 \mathrm{~V}$ \\
\hline 3) Rated output direct voltage & $V_{\text {out }}$ & $150 \mathrm{~V}$ \\
\hline 4) Switching frequency & $f_{\mathrm{s}}$ & $20 \mathrm{kHz}$ \\
\hline $\begin{array}{l}\text { 5) Absolute maximum ratings of } \\
\text { IGBT collector-emitter }\end{array}$ & $v_{\mathrm{CE}}$ & $600 \mathrm{~V}$ \\
\hline 6) On resistance of IGBT & $r_{\mathrm{t}}$ & $50 \mathrm{~m} \Omega$ \\
\hline 7) Absolute maximum ratings of diode & $i_{\mathrm{F}}$ & $30 \mathrm{~A}$ \\
\hline 8) Forward voltage of diode & $v_{\mathrm{F}}$ & $0.8 \mathrm{~V}$ \\
\hline 9) Recovery time of diode & $t_{\mathrm{rr}}$ & $0.1 \mu \mathrm{s}$ \\
\hline
\end{tabular}

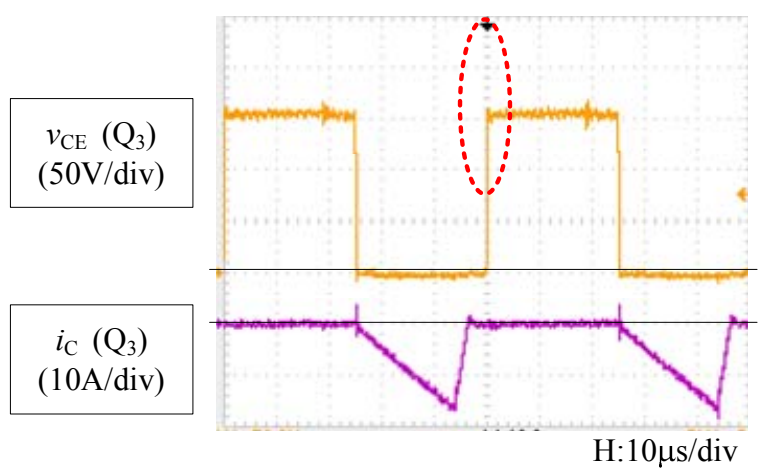

(b) the proposed operation

Figure 12. The waveforms in buck mode: (a) the conventional operation $\left(V_{\mathrm{in}}=200 \mathrm{~V}, V_{\text {out }}=150 \mathrm{~V}, P_{\mathrm{o}}=504 \mathrm{~W}\right)$;

(b) the proposed operation. $\left(V_{\text {in }}=200 \mathrm{~V}, V_{\text {out }}=150 \mathrm{~V}, P_{\mathrm{o}}=502 \mathrm{~W}\right)$

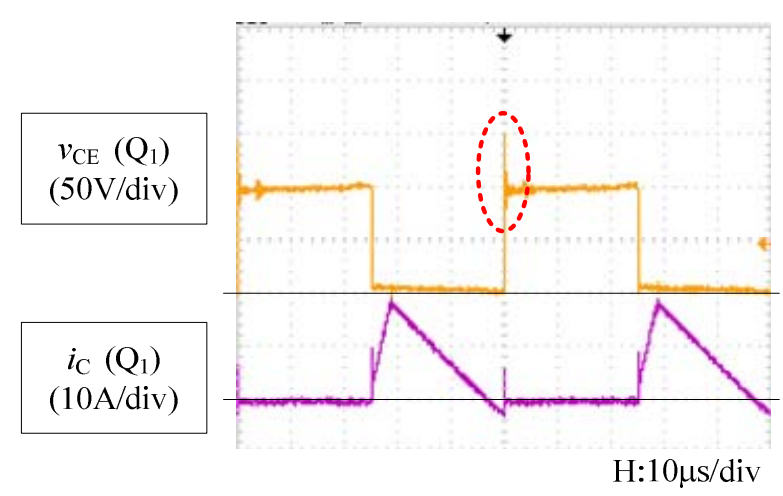

(a) the conventional operation

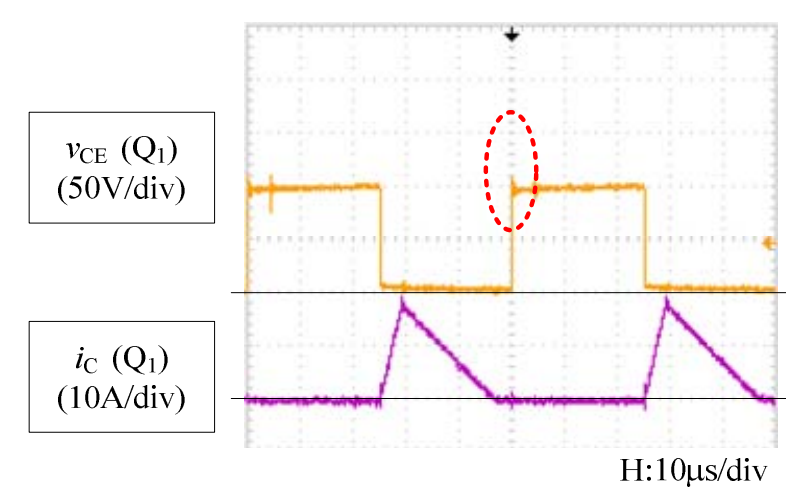

(b) the proposed operation

Figure 13. The waveforms in boost mode: (a) the conventional operation $\left(V_{\text {in }}=100 \mathrm{~V}, V_{\text {out }}=150 \mathrm{~V}, P_{\mathrm{o}}=687 \mathrm{~W}\right)$;

(b) the proposed operation. $\left(V_{\text {in }}=100 \mathrm{~V}, V_{\text {out }}=150 \mathrm{~V}, P_{\mathrm{o}}=686 \mathrm{~W}\right)$ 
of the can be decreased with the proposed operation. With these results, the ohmic loss at the parasitic resistor is also decreased. Fig. 16 shows the power efficiency results for the both of the conventional and the proposed operation. It can be seen that the power efficiency of buck mode can be apparently improved by up to $37 \%$ using the proposed operation at $100 \mathrm{~W}$ as shown in Fig. 16 (a). It can be seen that the power efficiency of boost mode can be apparently improved by up to $30 \%$ at $100 \mathrm{~W}$ as shown in Fig. 16 (b).

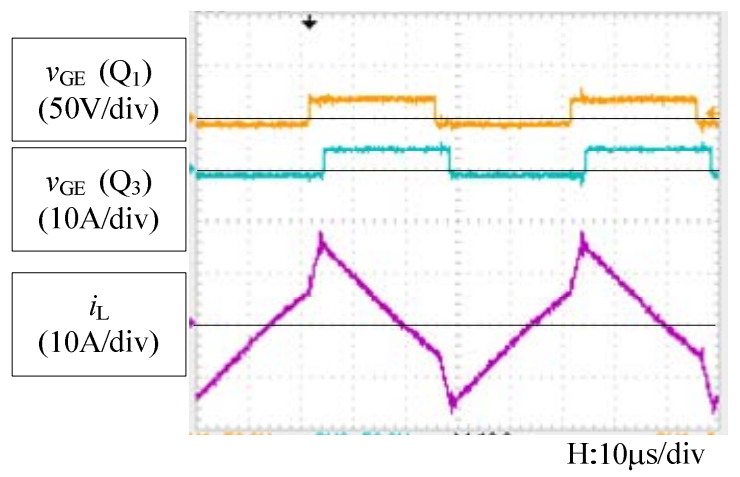

(a) the conventional operation

\section{Estimating the Value of Loss}

Fig. 17 shows $\varphi-P_{\mathrm{o}}$ of analysis and experimental results. For the analysis, $r_{\text {loss }}$ is estimated. $r_{\text {loss }}$ is set $1.5 \Omega$ for the conventional operation and $r_{\text {loss }}$ is set $0.5 \Omega$ for the proposed operation. This difference of $r_{\text {loss }}$ is due to the differences of the current path. Using these $r_{\text {loss }}$ values for both of them, differences between loss including analysis and experimental results are $10 \%$ or less in more than $100 \mathrm{~W}$. In less than $100 \mathrm{~W}$, error is greater than $10 \%$ because of measuring preci-

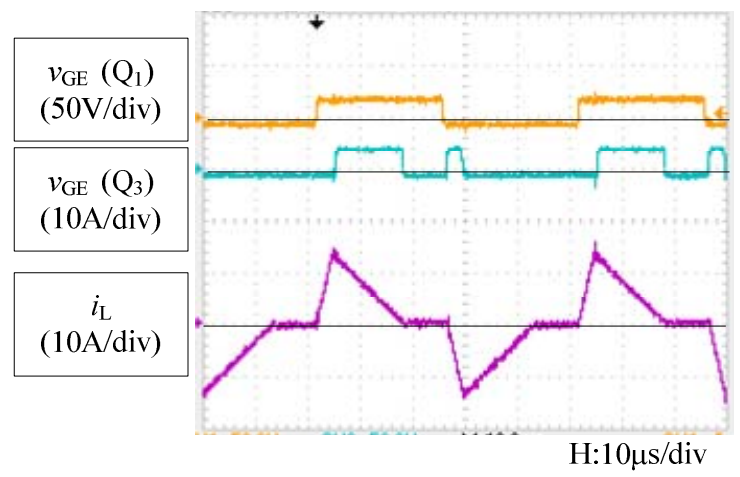

(b) the proposed operation

Figure 14. The comparison of the current waveforms of $i_{\mathrm{L}}$ : (a) the conventional operation $\left(V_{\text {in }}=100 \mathrm{~V}, V_{\text {out }}=150 \mathrm{~V}\right.$, $\left.P_{\mathrm{o}}=200 \mathrm{~W}\right)$; (b) the proposed operation $\left(V_{\text {in }}=100 \mathrm{~V}, V_{\text {out }}=150 \mathrm{~V}, P_{\mathrm{o}}=200 \mathrm{~W}\right)$.

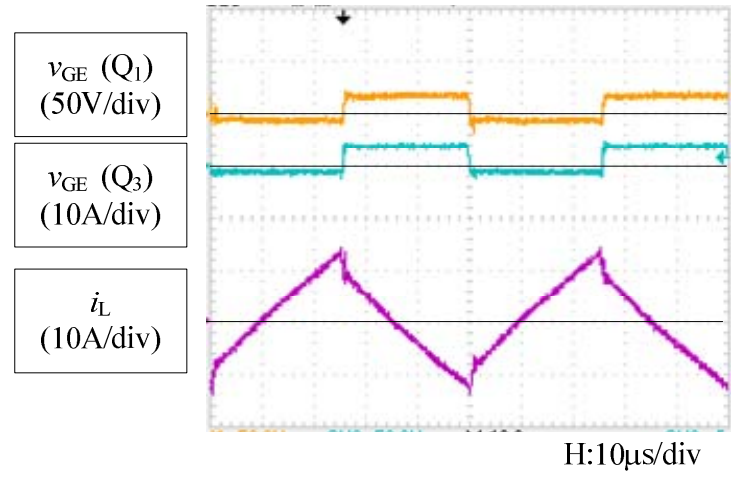

(a) the conventional operation

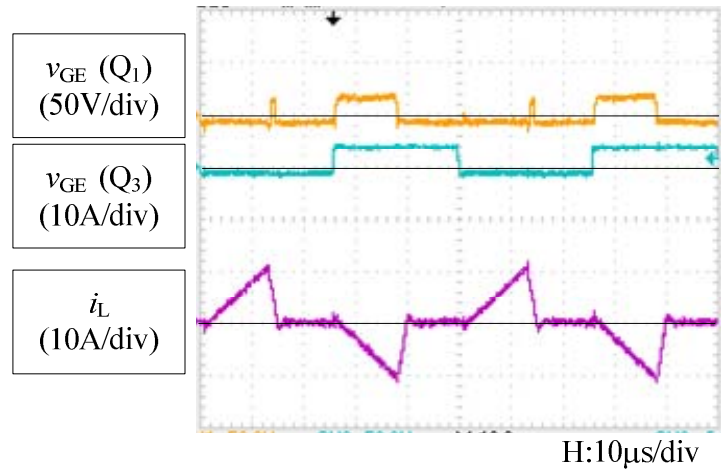

(b) the proposed operation

Figure 15. The comparison of the current waveforms of $i_{\mathrm{L}}$ : (a)the conventional operation $\left(V_{\text {in }}=200 \mathrm{~V}, V_{\text {out }}=150 \mathrm{~V}\right.$, $\left.P_{\mathrm{o}}=200 \mathrm{~W}\right)$; (b) the proposed operation $\left(V_{\text {in }}=200 \mathrm{~V}, V_{\text {out }}=150 \mathrm{~V}, P_{\mathrm{o}}=191 \mathrm{~W}\right)$.

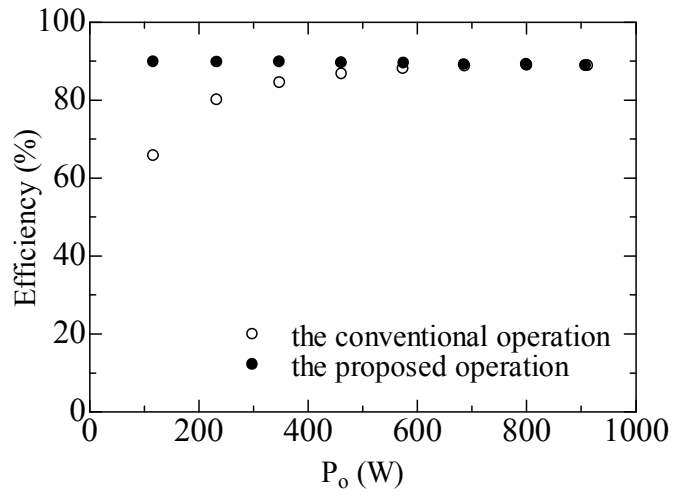

(a) buck mode

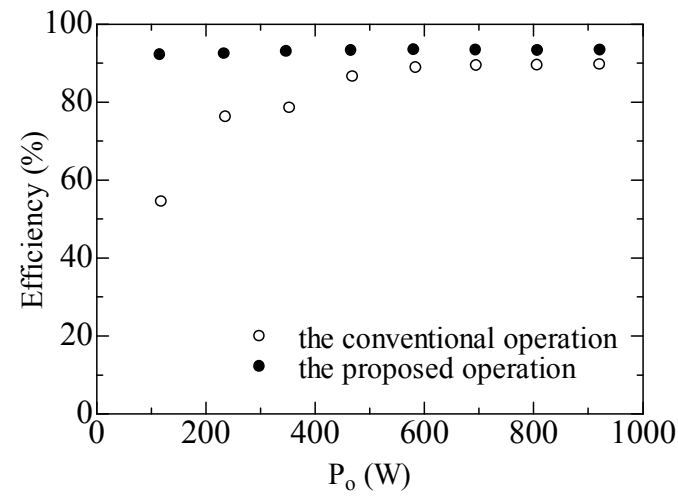

(b) boost mode

Figure 16. Power efficiency: (a) buck mode $\left(V_{\text {in }}=200 \mathrm{~V}, V_{\text {out }}=150 \mathrm{~V}\right)$; (b) boost mode $\left(V_{\text {in }}=100 \mathrm{~V}, V_{\text {out }}=150 \mathrm{~V}\right)$. 


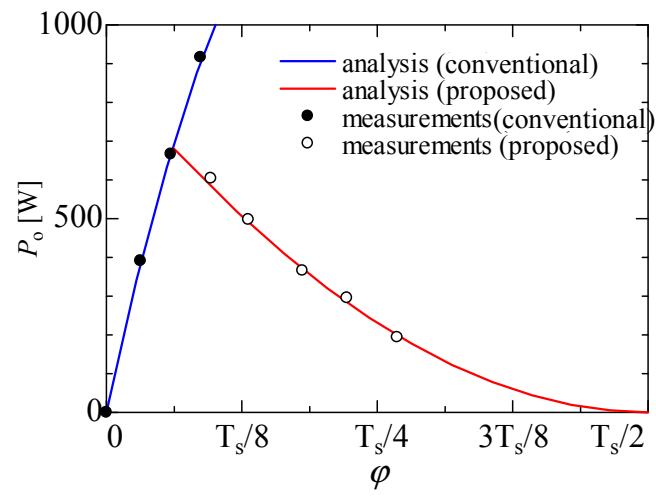

(a) buck mode

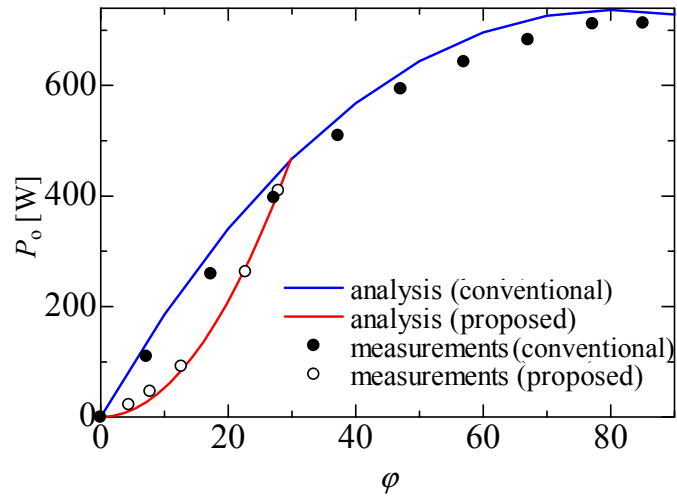

(b) boost mode

Figure 17. $\varphi-P_{\mathrm{o}}$ (analysis and experimental results).

sion. The results revealed that the analysis can be used to design the circuit.

\section{Compensation for floating terminalof transformer}

There is a state that the terminal of the transformer is open in the proposed operation. As the control option, it is possible to avoid the opening of the transformer by reducing the OFF time $(\mathrm{A} \sim \varphi, \mathrm{B} \sim \pi)$. One of the examples is shown in Fig. 18.

\section{CONCLUSION}

By the analysis of the circuit operation and the some experiments, the validation of the proposed operation for $\mathrm{DAB}$ dc-to-dc converter is revealed. Applying the two modes which are proposed operation in light load and conventional operation in heavy load, the circuit can be operated in the full load range. The operation method with digital operation can reduce switching surges without other circuits like snubber circuit. From the experiment results, the $99 \%$ of the maximum voltage surge reduction and $100 \%$ of the maximum current serge reduction at the light load is confirmed. Furthermore, due to the surge reduction method, $37 \%$ maximum power efficiency improvement can be confirmed at light load.

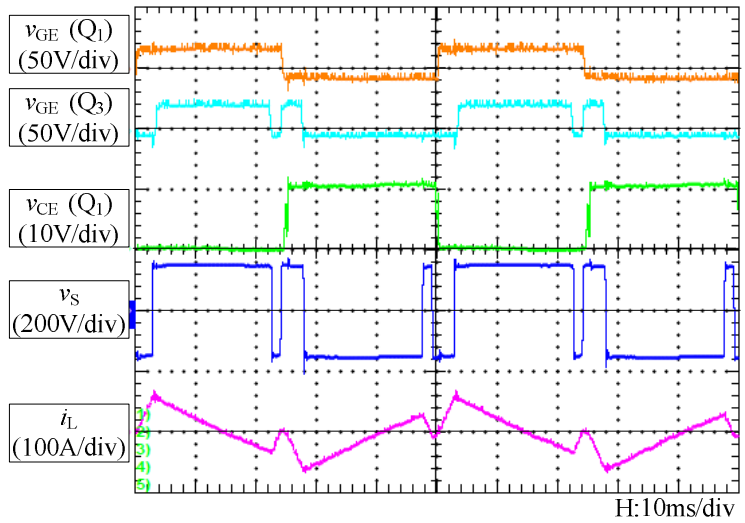

Figure 18. operation by reducing the OFF time.

$$
\left(V_{\text {in }}=100 \mathrm{~V}, V_{\text {out }}=150 \mathrm{~V}, P_{\mathrm{o}}=200 \mathrm{~W}\right)
$$

Furthermore $P_{\mathrm{o}}$ can be assumed with loss included analysis. Using $r_{\text {loss }}=1.5 \Omega$ at conventional operation and $r_{\text {loss }}=$ $0.5 \Omega$ at proposed operation, differences between loss including analysis and experimental results are $10 \%$ or less in more than $100 \mathrm{~W}$.

\section{ACKNOWLEDGMENT}

The authors would like to thank Mr. Shohei Iwasaki, technical staff, and Mr. Shun Nagata, Graduate Student, of Nagasaki University for the various technical assistances.

\section{REFERENCES}

[1] Mustansir H. Kheraluwala, Randal W. Gascoigne, Deepakraj M. Divan, and Eric D. Baumann, "Performance characterization of a high-power dual active bridge dc-to-dc converter," IEEE Trans. Industry Applications, vol.28, NO.6, pp. 1294-1301, Nov. / Dec. 1992

[2] Shigenori Inoue and Hirofumi Akagi, "A bidirectional isolated dc-dc converter as a core circuit of the next-generation medium-voltage power conversion system," IEEE Trans power Electron., vol.22, no.2, pp. 535-542, Mar. 2007.

[3] M. Pavlovsky, S. W. H. de Hann, and J. A. Ferreira, "Concept of $50 \mathrm{~kW}$ DC/DC converter based on ZVS, quasi-ZCS topology and integrated thermal and electronic design," 2005 European Conference on Power Electronics and Applications.

[4] Toshiro Hirose, Keisuke Nishimura, Takayuki Kimura, and Hirofumi Matsuo, "An AC-link Bidirectional DC-DC Converter with Synchronous Rectifier," in Proc. IECON, Nov. 2010.

[5] Rik W. A. A. De Doncker, Deepkraj M. Divan, and Mustansir H. Kheraluwala, "A Three-Phase Soft-Switched High-Power-Density $\mathrm{dc} / \mathrm{dc}$ Converter for High-Power Applications," IEEE Trans. Industry Applications, vol.27, NO.1, pp.63-73, Jan. / Feb. 1991

[6] N. Schibli, "Symmetrical multilevel converters with two quadrant DCDC feeding," Ph.D. dissertation, Swiss Federal Institute of Technology Lausanne (EPFL), 2000.

[7] Mika Takasaki, Yoichi Ishizuka, Tamotsu Ninomiya, Yutaka Furukawa, and Toshiro Hirose, "A Power Efficiency Improvement Technique for A Bi-Directional Dual Active Bridge DC-DC Converter at light load," Epe 2013 unpublished.

[8] Shun Nagata, Mika Takasaki, Kazuhide Domoto, Toshiro Hirose , and Yoichi Ishizuka, "A static characteristic analysis and high efficient technique of the light load condition of Bi-Directional Dual Active Bridge DC-DC Converter," IEICE EE, unpublished.

[9] Tamotsu Ninomiya, Masatoshi Nakahara, Toru Higashi, and Koosuke Harada, "A Unified Analysis of resonant Converters," IEEE Trans. Industry Applications, vol.27, NO.1, pp.63-73, Jan. / Feb. 1991 